\title{
Synchronized Progression of Prestin Expression and Auditory Brainstem Response during Postnatal Development in Rats
}

\author{
Jianfeng Hang, ${ }^{1,2}$ Wenlu Pan, ${ }^{1}$ Aoshuang Chang, ${ }^{1}$ Shun Li, ${ }^{3}$ \\ Cuixian Li, ${ }^{1}$ Mingyu Fu, ${ }^{1}$ and Jie Tang ${ }^{1}$ \\ ${ }^{1}$ Department of Physiology, School of Basic Medical Sciences, Southern Medical University, Guangzhou 510515, China \\ ${ }^{2}$ Department of Laboratory Medicines, General Hospital of Guangzhou Military Command of PLA, Guangzhou 510010, China \\ ${ }^{3}$ Affiliated High School of South China Normal University, Guangzhou 510630, China \\ Correspondence should be addressed to Jie Tang; jietang@smu.edu.cn
}

Received 8 September 2016; Revised 8 November 2016; Accepted 30 November 2016

Academic Editor: Jian Wang

Copyright (C) 2016 Jianfeng Hang et al. This is an open access article distributed under the Creative Commons Attribution License, which permits unrestricted use, distribution, and reproduction in any medium, provided the original work is properly cited.

\begin{abstract}
Prestin is the motor protein expressed in the cochlear outer hair cells (OHCs) of mammalian inner ear. The electromotility of OHCs driven by prestin is responsible for the cochlear amplification which is required for normal hearing in adult animals. Postnatal expression of prestin and activity of OHCs may contribute to the maturation of hearing in rodents. However, the temporal and spatial expression of prestin in cochlea during the development is not well characterized. In the present study, we examined the expression and function of prestin from the OHCs in apical, middle, and basal turns of the cochleae of postnatal rats. Prestin first appeared at postnatal day 6 (P6) for basal turn, P7 in middle turn, and P9 for apical turn of cochlea. The expression level increased progressively over the next few days and by P14 reached the mature level for all three segments. By comparison with the time course of the development of auditory brainstem response for different frequencies, our data reveal that prestin expression synchronized with the hearing development. The present study suggests that the onset time of hearing may require the expression of prestin and is determined by the mature function of OHCs.
\end{abstract}

\section{Introduction}

Mammalian hearing is characterized by incredible sensitivity and exquisite frequency selectivity. However, in many species including rodents, auditory function is developed after birth. In rats, action potential cannot be obtained from neurons in primary auditory cortex before postnatal days 10 to 11 (P10-P11) $[1,2]$. Therefore, this period at approximately P10 is an important stage of rat auditory development and is described as "hearing onset." During the following several days, the hearing sensitivity rapidly increases, and the minimal threshold for hearing decreases by approximately $30-$ $50 \mathrm{~dB}$ during P11-P14, reaching the adult level $[2,3]$. The maturation of auditory perception requires completion of the structural and functional development of both the auditory periphery and the central auditory system. Some important studies have investigated the maturation of central neurons and the refinement of the synaptic connections $[4,5]$. In vivo patch-clamp data show that subthreshold auditory inputs were observed in cortical neurons before hearing onset $[3,6]$. These reports suggest that cochlear function develops before hearing onset.

The morphological and functional development of the cochlea has been widely studied. Inner hair cells (IHCs) and outer hair cells (OHCs) are two types of sensory cells in the mammalian cochlea. OHCs convert sound vibration into electrical signals and change their cell length at acoustic frequencies $[7,8]$. These shape changes, termed electromotility, are assumed to be part of the mechanical feedback process that amplifies low-level sound $[7,9]$. The cochlear amplification derived from the motility of OHCs increases the sensitivity of weak sound by $40-60 \mathrm{~dB}[9,10]$. OHC electromotility is powered by a motor protein called prestin, which resides in the lateral membrane of OHCs. Driven by changes in membrane voltage, intracellular chloride ions move in or out of the prestin molecule, thereby triggering 
a switch in the conformation of prestin between the long and short states $[10,11]$. Interestingly, the morphological and functional maturation of OHCs occurs at postnatal ages $[12,13]$. Belyantseva et al. reported that prestin expression increases progressively after birth in a time course coinciding with that of electromotility [14]. These findings imply that the electromotility of IHCs is likely to be an important limiting factor in the development of mature hearing.

Auditory brainstem responses (ABRs) are likely an important manifestation of the global neural response to sound stimulation and are generally believed to be associated with in vivo hearing function. Therefore, in the present study, we investigated the development of the ABR and the expression of prestin in postnatal rats. Our results suggest that the expression and function of prestin coincide with the time course of ABR development.

\section{Materials and Methods}

2.1. Experimental Animals. Adult (2 to 3 months old) Sprague-Dawley rats and rat pups ranging from postnatal day 0 (P0) to P14 were used in this study. The animals were maintained on a 12-hour light/12-hour dark schedule and had free access to water and a standard diet. All of the studies were performed in accordance with the Chinese Prevention of Cruelty to Animals Act and permission was obtained from the Southern Medical University Laboratory Animal Center.

2.2. ABR Recording. Experiments were carried out on rat pups aged $\mathrm{P} 0-\mathrm{P} 14$ and on adult rats. Briefly, each animal was anesthetized with an intraperitoneal injection of sodium pentobarbital (22 mg/kg for pups and $30 \mathrm{mg} / \mathrm{kg}$ for adults). Then, the animal was placed on an antivibration table in a soundproof chamber. The animal's body temperature was maintained at $37.5^{\circ} \mathrm{C}$ with a heating pad during $\mathrm{ABR}$ recording. A subdermal needle electrode was placed over the skull vertex. The ground was placed ventrolateral to the left external pinna, and the reference was placed ventrolateral to the right external pinna. Calibrated stimuli were generated using TDT SigGenRP software (Tucker-Davis Technologies). Tone bursts ( $1 \mathrm{~ms}$ rise/fall, $3 \mathrm{~ms}$ plateau) of various frequencies $(1,2,4,8,16,24$, and $32 \mathrm{kHz})$ and intensities (0-90 dB SPL at $5 \mathrm{~dB}$ intervals) were presented using a calibrated TDT ES1 speaker located $50 \mathrm{~cm}$ away from the animal. The frequency-amplitude scan was computer controlled (TDT System 3, Tucker-Davis Technologies) and was delivered in a randomized sequence. Each frequencyamplitude combination was repeated 256 times at a rate of 10 bursts/s. The ABR signals were filtered $(100-1000 \mathrm{~Hz})$, amplified and averaged using TDT hardware (TDT System 3, Tucker-Davis Technologies), and recorded using BioSigRP software (Tucker-Davis Technologies). Hearing thresholds were determined by visual inspection of ABR waveforms and defined as the minimum intensity at which averaged waveforms could be distinguished. Data were stored for offline analysis. The entire recording for one animal spanned about 40 minutes.
2.3. Immunofluorescence Staining. The temporal bones of pups and adult Sprague-Dawley rats were isolated and placed into L-15 media (Invitrogen, Carlsbad, CA). The Organ of Corti was isolated and cut evenly into three pieces representing the basal, middle, and apical turns. Tissues were fixed with $4 \%$ paraformaldehyde in $0.1 \mathrm{M}$ PBS ( $\mathrm{pH} 7.4$ ) at $25^{\circ} \mathrm{C}$ for 2 hours. After being washed with PBS, the tissues were incubated in a blocking solution (10\% goat serum and $1 \%$ BSA in PBS) with $0.3 \%$ Triton X-100 for $20 \mathrm{~min}$ at room temperature and then incubated with a primary antibody against the prestin C-terminus (1:200, Santa Cruz Biotech Inc., Santa Cruz, CA) at $4^{\circ} \mathrm{C}$ overnight. After the primary antibodies were completely washed out with PBS, the tissue samples were incubated with Alexa Fluor 488-conjugated secondary antibodies (1:600; Invitrogen, Carlsbad, CA) in blocking solution at room temperature for $1 \mathrm{~h}$. Counterstaining of stereocilia was performed using phalloidin labeled with tetramethyl rhodamine isothiocyanate (TRITC-phalloidin, 1:200; Sigma, St. Louis, MO, USA) for $20 \mathrm{~min}$ at room temperature. After being washed three times in PBS, samples were mounted between a slide and a coverslip using ProLong antifade reagent (Invitrogen, Carlsbad, CA). Fluorescence images were obtained with a Nikon microscope (Nikon, A1+, Japan) using 20x or 40x objectives.

2.4. Western Blot. Western blot was performed as described in our previous study [15]. In brief, the apical turn of the Organ of Corti was isolated and homogenized in lysis buffer (50 mM Tris-HCl, pH 7.4, 150 mM NaCl, 1\% Triton X$100,1 \%$ sodium deoxycholate, and $0.1 \%$ SDS) supplemented with cOmplete ${ }^{\mathrm{TM}}$ protease inhibitor (Roche). After incubation on ice for $30 \mathrm{~min}$, samples were centrifuged at $12,000 \mathrm{rpm}$ for $15 \mathrm{~min}$ at $4^{\circ} \mathrm{C}$, and the supernatant was collected for further analysis. Subsequently, $20 \mu \mathrm{g}$ of total cochlear protein was separated using $8 \%$ SDS-PAGE and transferred to a nitrocellulose membrane (Millipore). After blocking with 5\% nonfat milk in TBS containing $0.1 \%$ Tween 20 , the membrane was incubated with primary antibodies (Sigma, St. Louis, MO, USA) overnight at $4^{\circ} \mathrm{C}$ on a shaking platform, followed by horseradish peroxidase-conjugated secondary antibodies (Invitrogen, Carlsbad, CA) for $1 \mathrm{~h}$ at room temperature. Immunoreactive bands were detected by enhanced chemiluminescence and visualized with the Amersham Imager $^{\mathrm{TM}} 600$ (GE Healthcare). Prestin and GAPDH bands were quantified using ImageQuant ${ }^{\mathrm{TM}}$ TL Image Analysis software (GE Healthcare) according to the manufacturer's instructions.

2.5. Patch-Clamp Recording and Nonlinear Capacitance Measurement. The animals were anesthetized by $\mathrm{CO}_{2}$ inhalation and decapitated. The cochlea was rapidly removed from the temporal bone and placed in a Petri dish filled with ice-cold Leibovitz's L-15 media (Invitrogen, Carlsbad, CA). The Organ of Corti was isolated from the apical turn of the cochlea and underwent mild enzymatic digestion with collagenase for $5 \mathrm{~min}$ ( $2 \mathrm{mg} / \mathrm{mL}$ collagenase IV, Sigma, St. Louis, MO). After gentle pipetting, the cells were transferred to a small plastic chamber filled with enzyme-free L-15 medium $(\sim 1.5 \mathrm{~mL})$ supplemented with 10 mM HEPES (pH 7.35 and 300 mOsm). 
The chamber then was placed on the stage of a Nikon inverted microscope (Eclipse Ti-S, Nikon, Japan) with a video camera (DS-Filc, Nikon, Japan). Healthy-appearing isolated OHCs were selected for the electrophysiological experiments. Cells showing any signs of shrinkage, swelling, or damage were excluded from the patch-clamp recording. Nonlinear capacitance (NLC) was measured as described in our previous publications [16-19]. The recording patch pipette was pulled by a pipette puller (P97, Sutter) and back-filled with the intracellular solution (140 CsCl, $10 \mathrm{EGTA}, 2 \mathrm{MgCl}_{2}$, and 10 HEPES in mM, $305 \mathrm{mOsm}$ and $\mathrm{pH}$ 7.35). Usually, the initial resistance of patch pipette was $2.5-3.5 \mathrm{M} \Omega$ in bath solution. Classical patch-clamp recording was performed under the whole-cell configuration using an Axopatch 200B amplifier and a $1440 \mathrm{~A} / \mathrm{D}$ converter (Molecular Devices, CA, USA). The membrane capacitance of OHCs was measured using a twosine-wave voltage stimulus protocol with a holding potential of $0 \mathrm{mV}$ [20]. The stimuli were controlled and the data were acquired using jClamp software (Scisoft, New Haven, CT). Software OriginPro (OriginLab Corporation, Northampton, MA) was used for offline analysis of the data. The NLC can be fitted with a two-state Boltzmann function and can reflect the nonlinear charge movements to membrane voltage [21]. The Boltzmann function for capacitance fitting is described as

$$
\begin{aligned}
& C_{m} \\
& =C_{\operatorname{lin}} \\
& \quad+\frac{Q_{\max } \alpha}{\exp \left[\alpha\left(V_{m}-V_{1 / 2}\right)\right]\left(1+\exp \left[-\alpha\left(V_{m}-V_{1 / 2}\right)\right]\right)^{2}} .
\end{aligned}
$$

Four parameters $\left(Q_{\max }, V_{1 / 2}, C_{\text {lin }}\right.$, and $\left.z\right)$ from the equation were related for the functional activity of prestin: $Q_{\max }$ is the maximum charge transfer across the membrane; $V_{1 / 2}$ (or $V_{h}$ ) is the membrane voltage at which the maximum charge movement occurs, or, equivalently, the peak of the capacitance-voltage function; $C_{\text {lin }}$ is the linear capacitance; and $\alpha$ represents the slope of the voltage dependence $(\alpha=$ $z e / k T$, where $k$ is the Boltzmann constant, $T$ is the absolute temperature, $z$ is the valence of the charge movement, and $e$ is the electron charge). $C_{\operatorname{lin}}$ is determined by the surface area of the cell membrane. To compare the magnitude values of the NLC obtained from OHCs with various cell sizes, we normalized NLC and $Q_{\max }$ to $C_{\text {lin }}$.

2.6. OHC Length Measurement. OHCs were isolated from the Organ of Corti as described above. In brief, the Organ of Corti was rapidly removed from the cochlea and cut evenly into three pieces in ice-cold L-15 medium. After mild enzymatic digestion for $5 \mathrm{~min}(2 \mathrm{mg} / \mathrm{mL}$ collagenase IV, Sigma, St. Louis, $\mathrm{MO})$, the tissues were transferred to a small plastic chamber filled with enzyme-free L-15 medium (7.35 pH, $300 \mathrm{mOsm})$. Hair cells were separated by gentle trituration of the tissue with a $100 \mu \mathrm{L}$ Hamilton syringe and a $25 \mathrm{G}$ needle. By using this technique, a fairly large number of isolated hair cells can be obtained. The chamber containing the hair cells was placed on the stage of a Nikon inverted microscope (Eclipse Ti-S, Nikon, Japan) equipped with a video camera (DS-Filc, Nikon,
Japan). Because of their morphology, in most preparations, IHCs and OHCs are easy to recognize. Usually, OHCs show a larger axis-diameter ratio, whereas IHCs have a tight neck [22]. The angle between the cuticular plate and the axis of the cell is another important landmark for identifying OHCs versus IHCs. Images of healthy-appearing solitary OHCs were captured using a video camera. The cell lengths of $\mathrm{OHCs}$ were measured offline.

2.7. Statistical Analysis. Results are presented as the mean \pm SD. Student's $t$-test was used to examine the significance of the difference between the data obtained from different postnatal ages. Significance was defined as $p<0.05$. The programs Excel and OriginPro were used for calculation, data fitting, and plotting.

\section{Results}

ABR waveforms were recorded from rat pups at various postnatal ages from day 0 (P0) to P14. During the first several days after birth, no ABR signal was observed, regardless of the frequency of sound stimulation and type of acoustic stimulus utilized (tone bursts, clicks, or noise). ABR for highfrequency tone bursts was detectable as early as P6. Responses were only found for stimuli of frequencies higher than $20 \mathrm{kHz}$ with relatively high intensity (thresholds $>75 \mathrm{~dB}$ SPL; 5 of 6 animals). Rapid changes occurred over the three subsequent days, and, by P9, the ABR could be readily identified for all frequencies tested. Figure 1(a) shows the representative ABR recordings to tone bursts at 1,16 , and $32 \mathrm{kHz}$ frequencies obtained from a rat pup at P9. Because wave I was the most consistent and robust component, it was used to estimate the ABR threshold (Figure 1(a), arrows). Thresholds for tone stimuli at different postnatal ages are shown in Figure 1(b). From P9 to P14, the ABR thresholds decreased progressively with increasing age, but the thresholds for different tone frequencies were affected differently. For frequencies higher than $24 \mathrm{kHz}$, the ABR responses were present as early as P6 in five of six animals. Mean thresholds $( \pm$ SD) in response to $32 \mathrm{kHz}$ tones at $\mathrm{P} 6, \mathrm{P} 7, \mathrm{P} 9, \mathrm{P} 11$, and $\mathrm{P} 14$ were $76.3 \pm 2.5$, $73.0 \pm 2.7,72.5 \pm 2.8,69.0 \pm 2.5$, and $51 \pm 2.2 \mathrm{~dB}$ SPL, respectively (Figure 1(c)), and the threshold at P14 did not differ significantly from that in adult animals $(47.5 \pm 5 \mathrm{~dB}$ SPL, $p>0.05, t$-test). ABRs for middle frequencies (4$16 \mathrm{kHz}$ ) were firstly observed at P7 in 7 of 7 animals. The mean thresholds $( \pm \mathrm{SD})$ in response to $16 \mathrm{kHz}$ tones at $\mathrm{P}$, $\mathrm{P} 9$, P11, and P14 were $74.0 \pm 2.2,67.5 \pm 2.8,62.5 \pm 2.8$, and $40 \pm 2.2 \mathrm{~dB}$ SPL, respectively (Figure 1(c)). The adult threshold $(17.1 \pm 2.6 \mathrm{~dB} \mathrm{SPL})$ was significantly lower than that observed at P14 $(p<0.01, t$-test). ABRs for low frequencies $(<2 \mathrm{kHz})$ appeared as late as $\mathrm{P} 9$. The mean thresholds $( \pm \mathrm{SD})$ in response to $1 \mathrm{kHz}$ tones at $\mathrm{P} 9, \mathrm{P} 11$, and $\mathrm{P} 14$ were $75 \pm 2.5,71.3 \pm 2.5$, and $63.4 \pm 2.7 \mathrm{~dB}$ SPL, respectively (Figure 1(c)), and thresholds at P14 did not differ from that in adults $(60.7 \pm 3.5 \mathrm{~dB}$ SPL, $p>0.05, t$-test). Our data show that, for most frequencies tested, the ABR thresholds at P14 were close to the adult levels. The arrows in Figure 1(c) indicate the first day when ABR appeared for different frequencies. 
$1 \mathrm{kHz}$

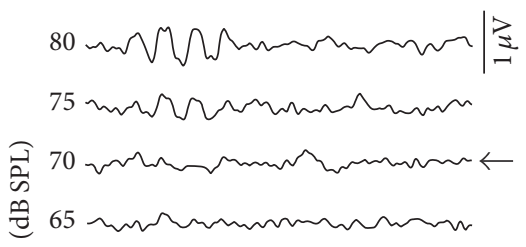

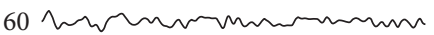

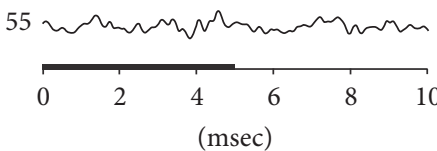

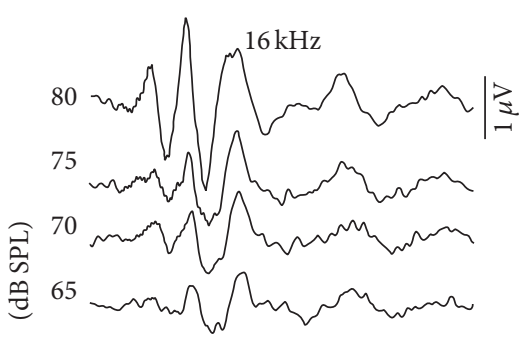

60 mann Snorme

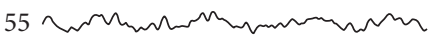

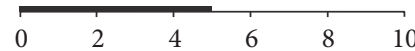

$32 \mathrm{kHz}$
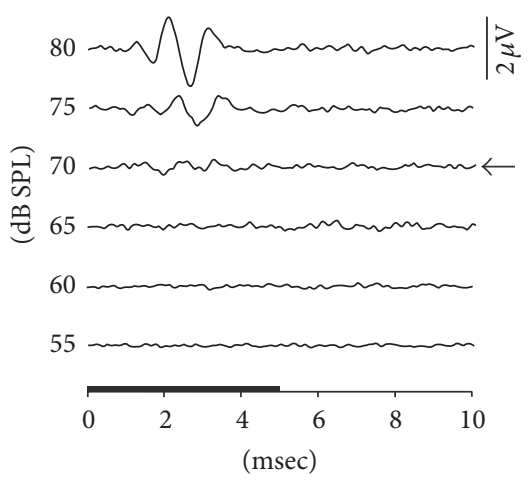

(a)

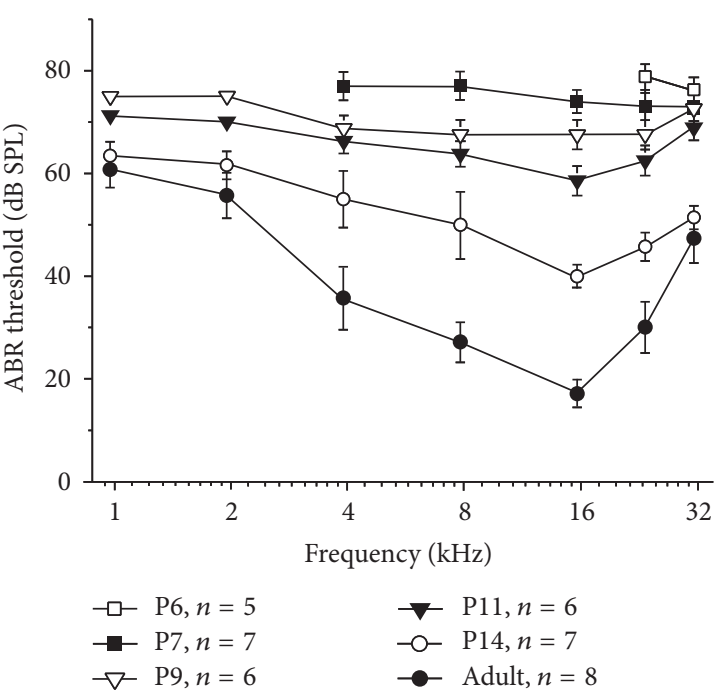

(b)

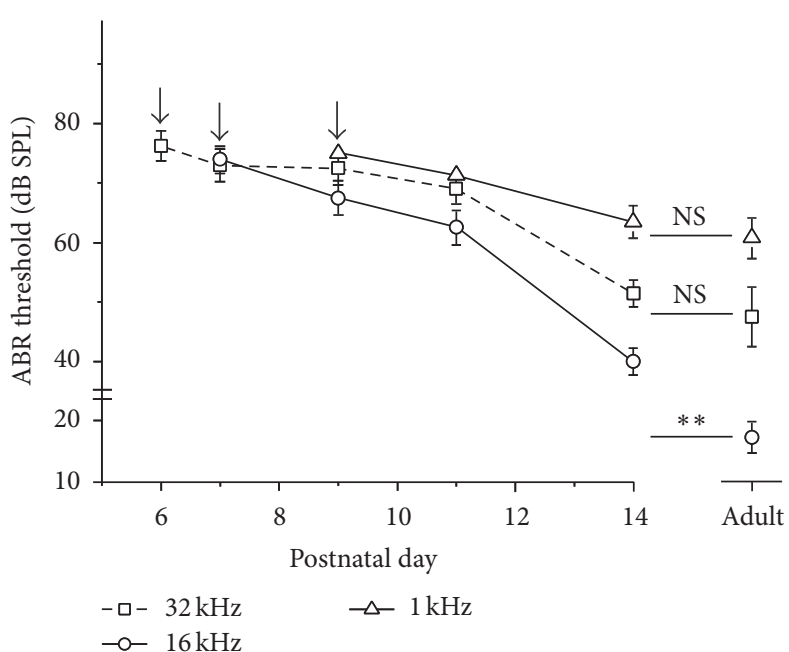

(c)

FIGURE 1: The ABR threshold changes during postnatal development. (a) The ABR waveforms recorded from a P9 rat pup. Arrows indicate ABR thresholds for $1 \mathrm{kHz}, 16 \mathrm{kHz}$, and $32 \mathrm{kHz}$ tones. The black horizontal bar on the $x$-axis denotes the 5 ms of the stimulus. (b) The ABR tuning curve changes during development. Data are expressed as the mean \pm SD. $n$ indicates the number of animals assessed. (c) The ABR thresholds decreased with time during postnatal development. Arrows indicate the first day on which the ABR appeared for different frequencies. ${ }^{* *} p<0.01 ;{ }^{\text {NS }} p>0.05$ (Student's $t$-test).

Because the waveform I of the ABR represents the response elicited from the cochlea, our data show that cochlear functions developed between P6 and P14. The electromotility of OHCs contributes significantly to the cochlear response. Our result implies that, during this period, the OHCs are very likely involved in the response to sound stimulation as the cochlear amplifier. The electromotility of OHCs is produced by the motor protein prestin, which is located on the lateral membrane. To investigate the density and position of prestin in the developing OHCs, immunolabeling of prestin was performed and images were collected along the length of cochlea by confocal microscopy. Figure 2 shows confocal micrographs of OHCs from the apical, middle, and basal turns of cochleae from P5 to P14 rat pups. For P5 and P7 apical turns, rhodamine-phalloidin staining for hair bundles is shown to define the apical surface of the OHCs. DAPI staining for nucleus is shown to indicate the bottom region of the OHCs. As shown in Figure 2, no prestin was detected in the OHCs along the entire cochlea at P5. Expression of prestin was first labeled at $\mathrm{P} 6$ to $\mathrm{P} 7$ in OHCs located in basal cochlea and showed a continued increase over the next week. Prestin expression progressed both spatially and temporally, with basal OHCs showing prestin expression $\sim 4$ days earlier than apical OHCs. By P14, robust ring-shaped fluorescence was observed in all three segments.

In order to quantify the observed expression level changes of prestin during development, we measured the immunoband intensity of prestin in apical OHCs at different postnatal ages by using western blotting. As shown by the representative immunobands in Figure 3(a), the expression of prestin increased in an age-dependent manner. Figure 3(b) shows the mean $( \pm S D)$ expression level from four independent experiments after normalization to GAPDH, the internal control protein. The expression of prestin remained 
Apical

P5
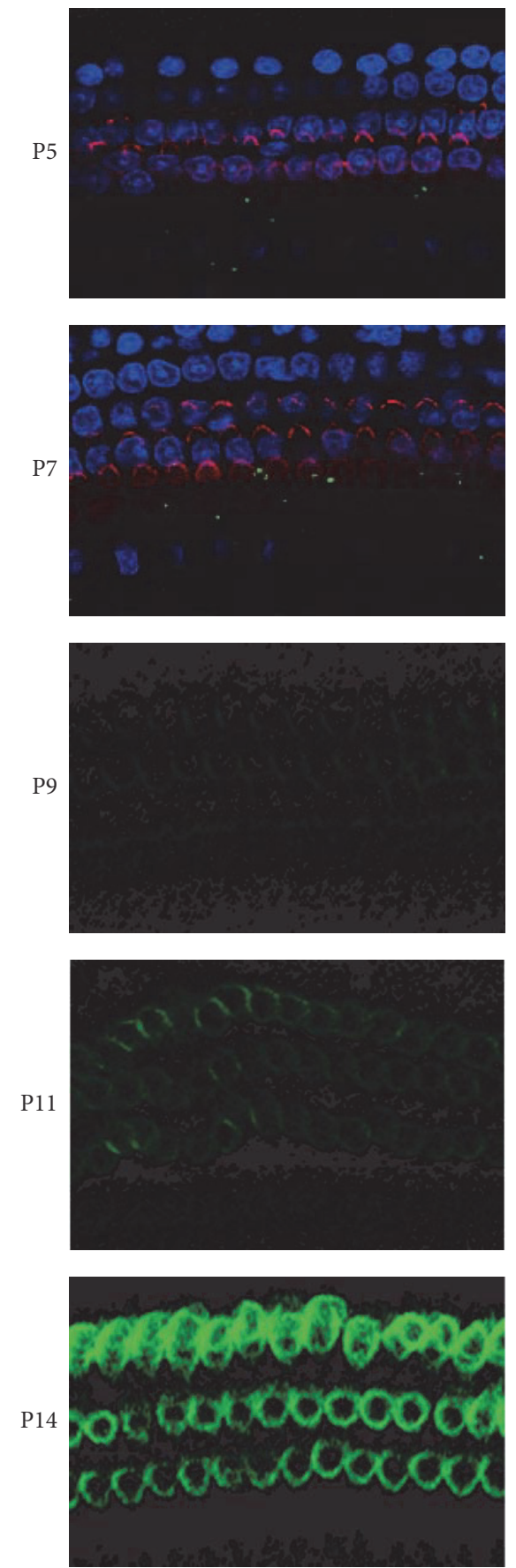

Middle
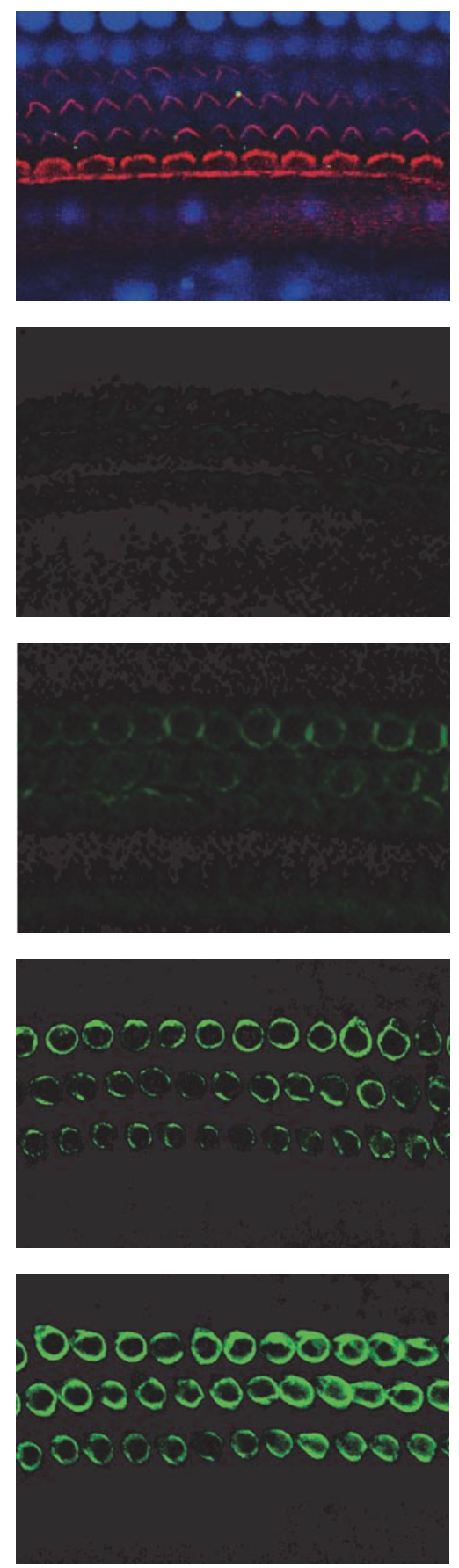

Basal
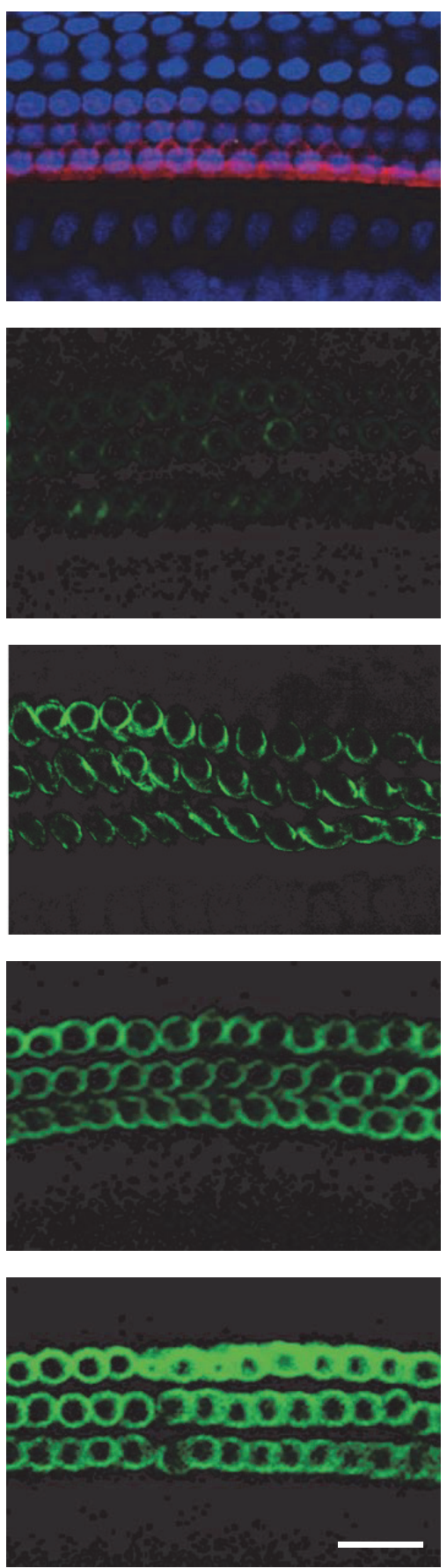

FIGURE 2: Confocal images of prestin expression in OHCs at various postnatal ages. Prestin immunoreactivity was measured from three segments (basal, middle, and apical) along the cochlea. For P5 and P7 apical turns, the hair bundles were labeled with rhodamine-phalloidin (red), the nuclei of hair cells were stained with DAPI (blue), and the prestin was labeled in green. Three images with different focal planes at hair bundle (to indicate the apical surface of the OHCs), the upper lateral wall of the HOCs, and nucleus were merged. No prestin fluorescence (green) was seen at P0-P5. To better illustrate the expression of prestin on the lateral wall of the OHCs, only green channel was shown after P7. Prestin expression progressed both spatially and temporally. Scale bar represents $20 \mu \mathrm{m}$.

low during the first five days after birth, and the most prominent increase in the prestin intensity occurred between P9 and P14. Although the prestin level increased slightly after P14, no significant difference was observed between the levels at P14 and adulthood.
Two essential electrophysiological properties are usually used to probe the function of prestin expressed in mammalian OHCs: NLC and electromotility. NLC and electromotility are fully coupled in mammals [21, 23, 24]. Because NLC can be easily and accurately measured experimentally, it 


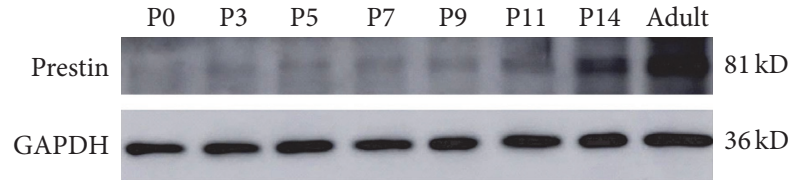

(a)

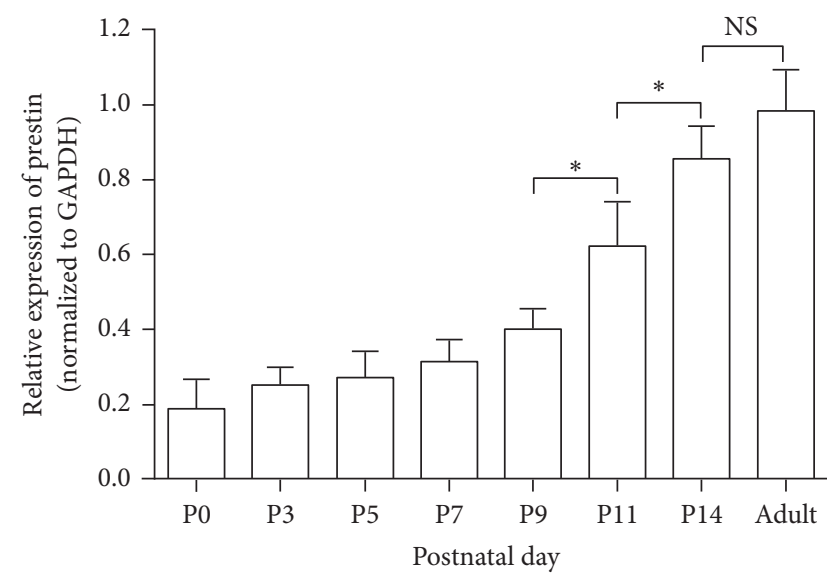

(b)

FIGURE 3: Expression of prestin in rats during cochlear development. (a) Representative prestin and GAPDH immunobands from western blots were detected at $81 \mathrm{kD}$ and $36 \mathrm{kD}$. GAPDH was used as the internal control. (b) Relative expression of prestin (all normalized to GAPDH) at different postnatal ages. Comparisons are shown by the bars above the relevant columns. Data were obtained from four independent experiments and are expressed as mean $\pm \mathrm{SD} .{ }^{*} p<0.05 ;{ }^{* *} p<0.01 ;{ }^{\mathrm{NS}} p>0.05$ (Student's $t$-test).

is used as an assay to evaluate the functional development of prestin in the experiment described below. In OHCs of adult rodents, NLC is characterized by bell-shaped dependence on membrane potential with a peak at approximately $-70 \mathrm{mV}$ [21]. As shown in Figure 4, we compared the NLC recorded from apical OHCs from P6 to P14 with adult OHCs. To eliminate the influence of different cell sizes, the magnitude of NLC was normalized to $C_{\text {lin }}$ (representing the surface area of OHC). Figure 4(a) shows that NLC responses were first detectable in 4 of 9 OHCs at P6, with very small magnitude (Figure 4(a), black line). Robust NLC responses were recorded in all OHCs measured from $\mathrm{P} 9$, and the responses increased throughout the developmental time period studied. At P14, the NLC reached a level comparable with that in adults. Parameters derived from the curve fitting with the two-state Boltzmann function are summarized in Figures 4(b)-4(e). It is apparent that $V_{1 / 2}$ during early development (P6) is relatively low $(-83.96 \pm 8.79 \mathrm{mV})$; however, at ages above P9, $V_{1 / 2}$ increases to the adult level $(-66.46 \pm 6.50 \mathrm{mV}$, Figure $4(\mathrm{~b}))$. $\mathrm{NLC} / \mathrm{C}_{\mathrm{lin}}$ and $\mathrm{Q}_{\max } / C_{\text {lin }}$ are both correlated with the moving charge density or with the amount of activated prestin. The larger the values, the more the charges translocated during voltage stimulation. As shown in Figures 4(c) and 4(d), both $\mathrm{NLC} / C_{\text {lin }}$ and $Q_{\max } / C_{\text {lin }}$ increase between P6 and P14, and these values reach maturity at P14. The voltage sensitivity or valence, $z$, remained stable at all ages studied (Figure 4(e), $p>0.05$, $t$-test).

Because electromotility occurs along the longitudinal axis of OHCs, it is generally accepted that OHC length is critical for cochlear amplification. With the identical prestin expression level and activity, the longer $\mathrm{OHCs}$ could generate more significant motility and therefore conduct larger cochlear amplification. We measured the cell length of OHCs isolated from different segments along the basilar membrane. Figure 5 shows that the OHC length kept increasing and exhibited a sigmoidal rise from $\mathrm{P} 0$ to $\mathrm{P} 14$. The length of basal OHCs had minor changes over this period while the apical OHCs kept growing until approximately P11. Basal, middle, and apical OHCs reached their adult lengths at P5, P8, and P11, respectively (indicated by stars in Figure 5), and apical OHCs matured later than middle and basal OHCs.

\section{Discussion}

In this study, we used the ABR to measure the hearing sensitivity in developing rat pups. The ABR is a compound evoked potential derived from the acoustic responses of different stages along the ascending pathway of the auditory system. It is generally accepted that the peaks of ABR waveforms with different time delay are generated by distinct brainstem nuclei [25]. The responses of the cochlea and the auditory nerve are primarily responsible for peaks I and II, respectively. Peaks III to V (up to seven peaks were observed in some studies) represent the responses from the cochlear nucleus and other brainstem nuclei in higher level. Compared with recordings from adult animals, our data show that the ABR waveforms obtained from pups younger than P14 showed some special features. Only three to four distinct waves were identifiable within the first $8 \mathrm{~ms}$ after stimulus onset, as shown in Figure 1. The latency of peak I is very similar to previously published examples from adults (e.g., $1.4 \pm 0.24 \mathrm{~ms}$ in P9 pups versus $1.2 \pm 0.15 \mathrm{~ms}$ in adults for $32 \mathrm{kHz}, 90 \mathrm{~dB}$ SPL tones) [26]. Therefore, our results suggest that the acoustic responses of higher level auditory nuclei are absent in developing rats. Although we did not examine the development of responses for individual nuclei in the central auditory system, our results imply that central auditory neurons reach functional maturity later than do cochleae and the cochlear signal inputs are required for the development of the auditory system. This hypothesis is supported by recent evidence from in vivo patch-clamp recording from cortical auditory neurons. The excitatory and inhibitory synaptic inputs are not cotuned 

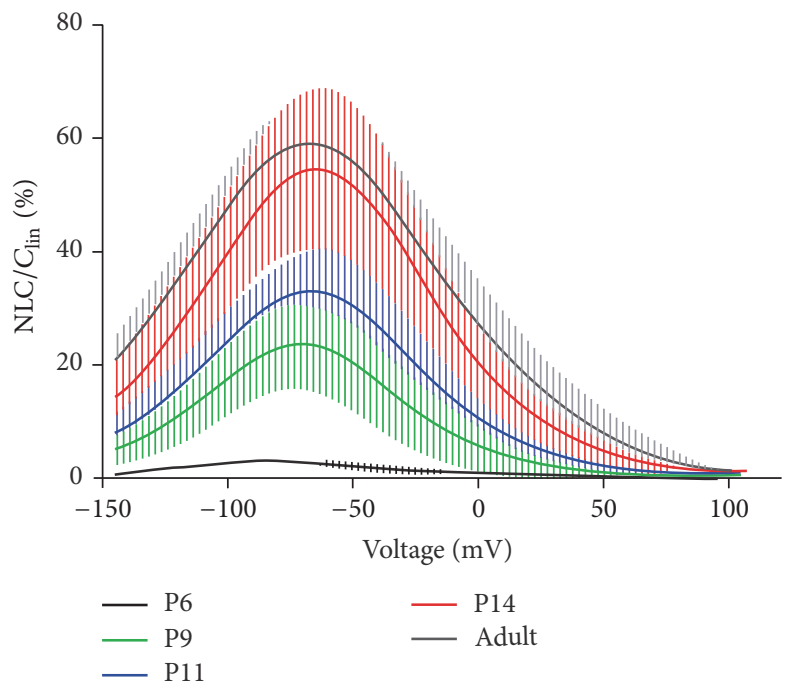

(a)

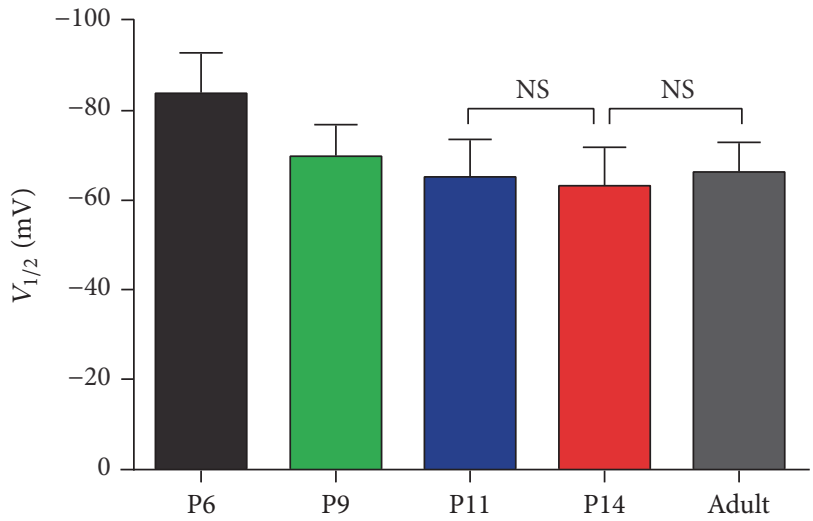

(b)

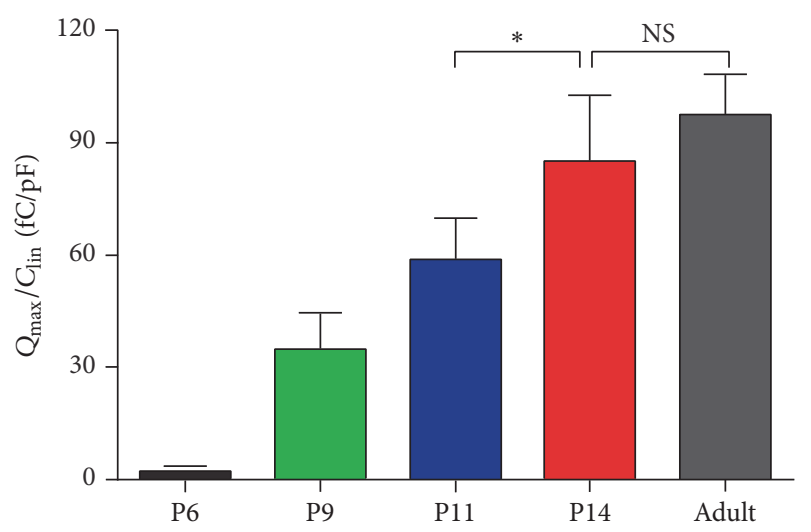

(d)

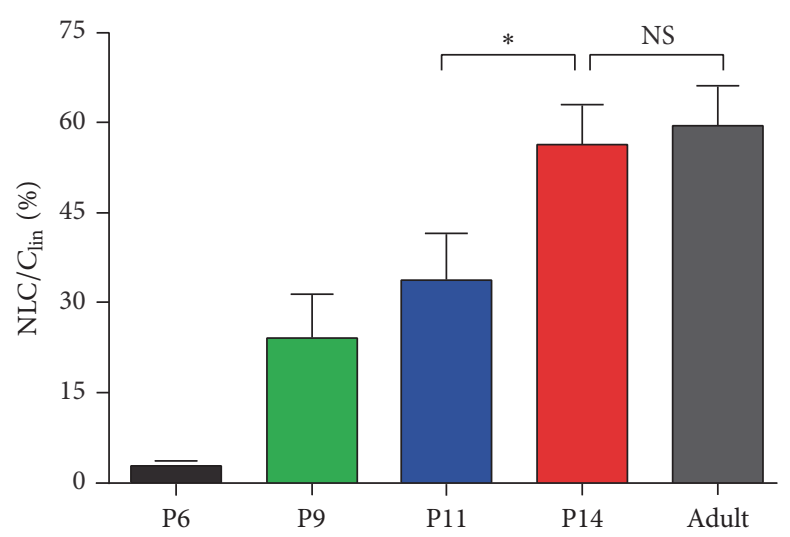

(c)

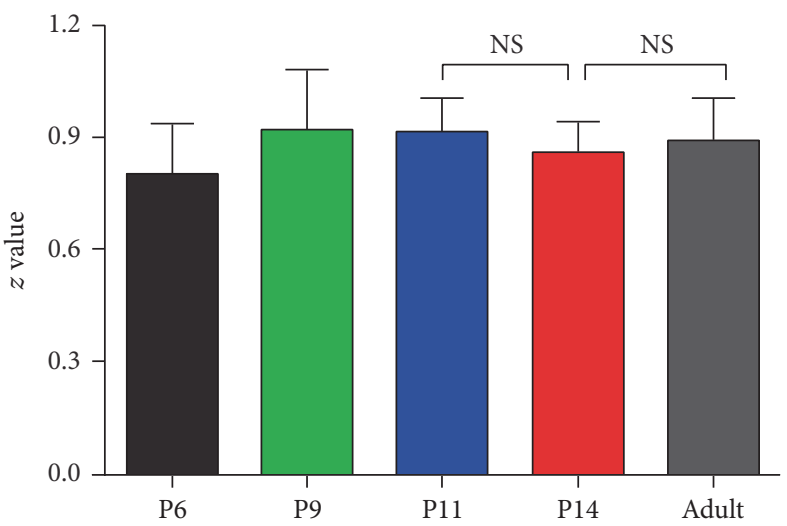

(e)

FIGURE 4: NLC measured from apical OHCs at various postnatal ages. (a) The mean NLC-voltage responses were fitted with the Boltzmann function (color coded heavy lines). NLC was normalized by $C_{\text {lin }}$ and the curves were plotted as the mean \pm SD. ((b)-(e)) Four parameters derived from curve fitting with Boltzmann's function. See results for details. Data are expressed as the mean \pm SD. $n=4,8,8,12$, and 10 for P6, P9, P11, P14, and adult, respectively. ${ }^{*} p<0.05 ;{ }^{\text {NS }} p>0.05$ (Student's $t$-test). 


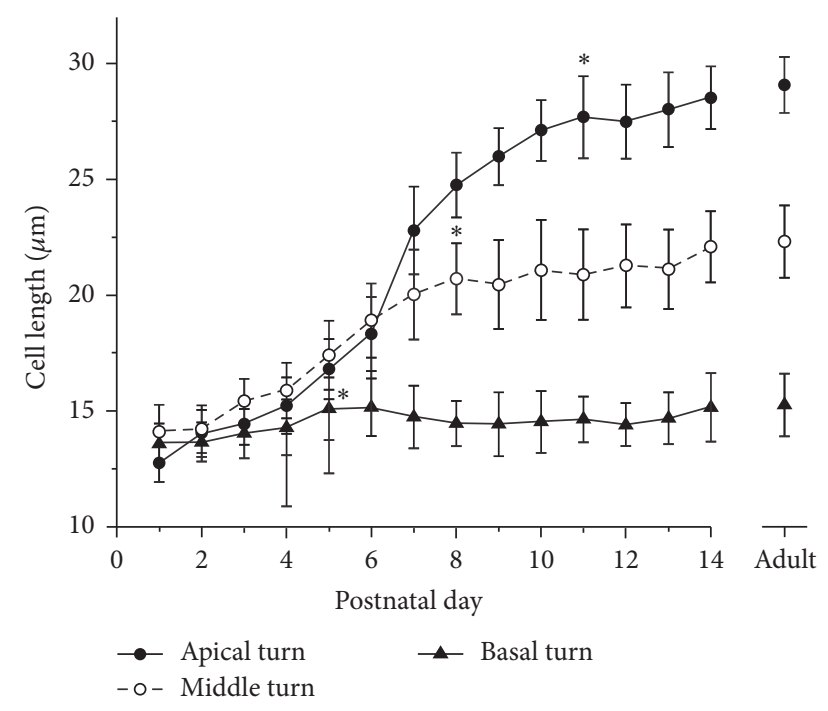

FIGURE 5: Changes in OHC length as a function of postnatal day. Each data point is plotted as the mean and SD of at least 50 OHCs. Stars indicate the earliest day at which cells reach the adult length ( $p>0.05$, Student's $t$-test).

and are temporally imbalanced before P12; therefore, action potentials were rarely recorded before hearing onset $[3,6]$. These results suggest that the onset of cochlear responses occurs earlier than the synaptic refinement in the auditory cortex. Our results also show that the ABR response is detectable as early as P6-P9, although the time line is various for different frequencies (Figure 1).

However, the mechanisms responsible for the onset of the cochlear response and for determining the maturation time of cochlear function remain unclear. It is clear that substantial morphological and functional changes occur during cochlear development. In rats, the structure of the cochlea is formed at an early stage after birth; the middle ear is well developed and the ear canal is open before hearing onset [27-29]. The differentiation of hair cells and supporting cells, as well as the innervation of spiral ganglion neurons, is completed during the embryonic period [13, 3035]. The morphological development of hair bundles and the functional maturation of mechanoelectrical transduction are completed before postnatal day $6[13,28,29,36,37]$. Because the cochlear response begins to appear at P6-P9 according to our ABR waveform analysis, the above processes are not likely to determine the onset of the cochlear response. In rats, action potentials of cortical neurons in response to sound stimulation are first recordable at P10-P11. Responses were found only for relatively high-intensity stimuli. Rapid changes occurred over the 3 subsequent days, and, by P14, the auditory cortex showed adult-like excitatory responses. The most significant change is the $40-50 \mathrm{~dB}$ threshold decreasing $[1,2]$. This dramatic change in hearing sensitivity implies the involvement of a mechanism that modulates the sensitivity of the hearing system during this period.

Cochlear amplification confers extremely high sensitivity to our hearing with an enormous intensity range [38].
Mammalian cochlear amplification is attributed to OHCs, which can change their cell length in response to changes in membrane potential synchronized to sound waves [7]. This activity termed electromotility is powered by the unique motor protein prestin in the $\mathrm{OHC}$ lateral membrane [11]. Our study indicates that prestin is first expressed at P6 (Figure 2) and reaches the adult expression level at P14 (Figures 3 and 4). This period closely corresponds to the onset of cochlear function (Figure 1). The developmental expression pattern of prestin has been studied in two species, rat and mouse [14, 39]. Belyantseva et al. reported that prestin expression begins at postnatal day 0 and increases progressively in a time course coinciding with that of electromotility [14]. Our results do not appear to be consistent with these data. Expression of prestin in OHCs from different positions within the cochlea (basal, middle, and apical turns) is not synchronized: it reached the level of maturity in the basal turn at P9, in the middle turn at P10-P11, and in the apical turn at P12 (Figures 2 and 3). Our results are supported by function measurements of OHCs in other species. In mouse and gerbil, the NLC and electromotility of OHCs are first detected at P6 and reach adult levels at P12-P15 [12, 39]. In rats, however, the expression of prestin and the electromotility of OHCs are synchronous during development.

In the present study, we did not measure the motility of OHCs directly. NLC and electromotility are fully coupled in mammalian OHCs $[21,23,24]$ and the former could be evaluated accurately [16-19]. Our NLC measurements show that, for apical OHCs, the function of prestin first appears at P9 and reached mature level at P14 (Figure 4). This result is consistent with the data recorded from mouse [39]. This functional measurement is also concordant with our immunofluorescence staining and western blot results (Figures 2 and 3). Thus, we proposed that the development of auditory central neural system, indicated by an increased sensitivity of ABR responses, occurs as the functional maturation of prestin, the motor protein of the OHCs.

Besides the expression level and functional activity, the cell length of OHCs is definitely an important factor that affects the overall motility magnitude of OHCs. As shown in Figure 5, the times at which mature cell lengths were reached are P5, P8, and P11 for basal, middle, and apical OHCs, respectively. These time points are earlier than those of the prestin expression. Therefore, we conclude that cell length development is not likely the limiting factor to determine the onset time of cochlear function. It is well established that the basal segments of the cochlea respond to highfrequency stimuli while more apical portions are tuned to low frequencies. Our data revealed that the spatial and temporal expression patterns of prestin and development of auditory brainstem response are highly concordant. It is more likely that the expression and functional development of prestin may play a critical role in the maturity of hearing.

\section{Conclusions}

In conclusion, our results indicate that the expression pattern of prestin and the NLC function of OHCs are synchronized 
with the development of auditory brainstem responses. This finding suggests that the expression and functional development of prestin may determine the onset of hearing of rats.

\section{Competing Interests}

The authors declare that they have no competing interests.

\section{Acknowledgments}

This work was supported by grants from the 973 Program (2014CB943002) and the National Natural Science Foundation of China (31271179, 31500841).

\section{References}

[1] L. I. Zhang, S. Bao, and M. M. Merzenich, "Persistent and specific influences of early acoustic environments on primary auditory cortex," Nature Neuroscience, vol. 4, no. 11, pp. 11231130, 2001.

[2] E. de Villers-Sidani, E. F. Chang, S. Bao, and M. M. Merzenich, "Critical period window for spectral tuning defined in the primary auditory cortex (A1) in the rat," The Journal of Neuroscience, vol. 27, no. 1, pp. 180-189, 2007.

[3] Y. J. Sun, G. K. Wu, B.-H. Liu et al., "Fine-tuning of prebalanced excitation and inhibition during auditory cortical development," Nature, vol. 465, no. 7300, pp. 927-931, 2010.

[4] D. H. Sanes and S. Bao, "Tuning up the developing auditory CNS," Current Opinion in Neurobiology, vol. 19, no. 2, pp. 188199, 2009.

[5] R. C. Froemke and B. J. Jones, "Development of auditory cortical synaptic receptive fields," Neuroscience \& Biobehavioral Reviews, vol. 35, no. 10, pp. 2105-2113, 2011.

[6] A. L. Dorrn, K. Yuan, A. J. Barker, C. E. Schreiner, and R. C. Froemke, "Developmental sensory experience balances cortical excitation and inhibition," Nature, vol. 465, no. 7300, pp. 932936, 2010.

[7] W. E. Brownell, C. R. Bader, D. Bertrand, and Y. De Ribaupierre, "Evoked mechanical responses of isolated cochlear outer hair cells," Science, vol. 227, no. 4683, pp. 194-196, 1985.

[8] P. Dallos, B. N. Evans, and R. Hallworth, "Nature of the motor element in electrokinetic shape changes of cochlear outer hair cells," Nature, vol. 350, no. 6314, pp. 155-157, 1991.

[9] P. Dallos, "The active cochlea," The Journal of Neuroscience, vol. 12, no. 12, pp. 4575-4585, 1992.

[10] P. Dallos, "Cochlear amplification, outer hair cells and prestin," Current Opinion in Neurobiology, vol. 18, no. 4, pp. 370-376, 2008.

[11] J. Zheng, W. Shen, D. Z. Z. He, K. B. Long, L. D. Madison, and P. Dallos, "Prestin is the motor protein of cochlear outer hair cells," Nature, vol. 405, no. 6783, pp. 149-155, 2000.

[12] D. Z. Z. He, B. N. Evans, and P. Dallos, "First appearance and development of electromotility in neonatal gerbil outer hair cells," Hearing Research, vol. 78, no. 1, pp. 77-90, 1994.

[13] J. Waguespack, F. T. Salles, B. Kachar, and A. J. Ricci, "Stepwise morphological and functional maturation of mechanotransduction in rat outer hair cells," The Journal of Neuroscience, vol. 27, no. 50, pp. 13890-13902, 2007.

[14] I. A. Belyantseva, H. J. Adler, R. Curi, G. I. Frolenkov, and B. Kachar, "Expression and localization of prestin and the sugar transporter GLUT-5 during development of electromotility in cochlear outer hair cells," The Journal of Neuroscience, vol. 20, no. 24, Article ID RC116, 2000.

[15] C. Li, S. Chen, Y. Yu et al., "BIG1, a brefeldin A-inhibited guanine nucleotide-exchange factor, is required for GABA-gated $\mathrm{Cl}^{-}$ influx through regulation of $\mathrm{GABA}_{A}$ receptor trafficking," Molecular Neurobiology, vol. 49, no. 2, pp. 808-819, 2014.

[16] X. Tan, J. L. Pecka, J. Tang et al., "From zebrafish to mammal: functional evolution of prestin, the motor protein of cochlear outer hair cells," Journal of Neurophysiology, vol. 105, no. 1, pp. 36-44, 2011.

[17] X. Tan, J. L. Pecka, J. Tang, S. Lovas, K. W. Beisel, and D. Z. Z. $\mathrm{He}$, "A motif of eleven amino acids is a structural adaptation that facilitates motor capability of eutherian prestin," Journal of Cell Science, vol. 125, part 4, pp. 1039-1047, 2012.

[18] J. Tang, J. L. Pecka, X. Tan, K. W. Beisel, and D. Z. Z. $\mathrm{He}$, "Engineered pendrin protein, an anion transporter and molecular motor," The Journal of Biological Chemistry, vol. 286, no. 35, pp. 31014-31021, 2011.

[19] J. Tang, J. L. Pecka, B. Fritzsch, K. W. Beisel, and D. Z. Z. He, "Lizard and frog prestin: evolutionary insight into functional changes," PLoS ONE, vol. 8, no. 1, Article ID e54388, 2013.

[20] J. Santos-Sacchi, M. Wu, and S. Kakehata, "Furosemide alters nonlinear capacitance in isolated outer hair cells," Hearing Research, vol. 159, no. 1-2, pp. 69-73, 2001.

[21] J. Santos-Sacchi, "Reversible inhibition of voltage-dependent outer hair cell motility and capacitance," The Journal of Neuroscience, vol. 11, no. 10, pp. 3096-3110, 1991.

[22] D. Z. Z. He, J. Zheng, R. Edge, and P. Dallos, "Isolation of cochlear inner hair cells," Hearing Research, vol. 145, no. 1-2, pp. 156-160, 2000.

[23] J. F. Ashmore, Cochlear Mechanisms: Structure, Function, and Models, Springer Science \& Business Media, 1989.

[24] K. Homma and P. Dallos, "Evidence that prestin has at least two voltage-dependent steps," The Journal of Biological Chemistry, vol. 286, no. 3, pp. 2297-2307, 2011.

[25] A. Møller, Hearing: Anatomy, Physiology, and Disorders of the Auditory System, Elsevier, Oxford, UK, 2006.

[26] P. Scimemi, R. Santarelli, A. Selmo, and F. Mammano, "Auditory brainstem responses to clicks and tone bursts in C57 BL/6j mice," Acta Otorhinolaryngologica Italica, vol. 34, no. 4, pp. 264271, 2014.

[27] M. Geal-Dor, S. Freeman, G. Li, and H. Sohmer, "Development of hearing in neonatal rats: air and bone conducted ABR thresholds," Hearing Research, vol. 69, no. 1-2, pp. 236-242, 1993.

[28] M. C. Kelly and P. Chen, "Development of form and function in the mammalian cochlea," Current Opinion in Neurobiology, vol. 19, no. 4, pp. 395-401, 2009.

[29] A. K. Groves and D. M. Fekete, "Shaping sound in space: the regulation of inner ear patterning," Development, vol. 139, no. 2, pp. 245-257, 2012.

[30] M. W. Kelley, "Regulation of cell fate in the sensory epithelia of the inner ear," Nature Reviews Neuroscience, vol. 7, no. 11, pp. 837-849, 2006.

[31] J. M. Appler and L. V. Goodrich, "Connecting the ear to the brain: molecular mechanisms of auditory circuit assembly," Progress in Neurobiology, vol. 93, no. 4, pp. 488-508, 2011.

[32] J. Defourny, F. Lallemend, and B. Malgrange, "Structure and development of cochlear afferent innervation in mammals," American Journal of Physiology-Cell Physiology, vol. 301, no. 4, pp. C750-C761, 2011. 
[33] C. C. Lu, J. M. Appler, E. A. Houseman, and L. V. Goodrich, "Developmental profiling of spiral ganglion neurons reveals insights into auditory circuit assembly," The Journal of Neuroscience, vol. 31, no. 30, pp. 10903-10918, 2011.

[34] T. M. Coate, S. Raft, X. Zhao, A. Ryan, E. B. Crenshaw, and M. W. Kelley, "Otic mesenchyme cells regulate spiral ganglion axon fasciculation through a Pou3f4/EphA4 signaling pathway," Neuron, vol. 73, no. 1, pp. 49-63, 2012.

[35] S.-Z. Wang, L. A. Ibrahim, Y. J. Kim et al., "Slit/Robo signaling mediates spatial positioning of spiral ganglion neurons during development of cochlear innervation," The Journal of Neuroscience, vol. 33, no. 30, pp. 12242-12254, 2013.

[36] A. Dabdoub, M. J. Donohue, A. Brennan et al., "Wnt signaling mediates reorientation of outer hair cell stereociliary bundles in the mammalian cochlea," Development, vol. 130, no. 11, pp. 2375-2384, 2003.

[37] P. C. G. Rida and P. Chen, "Line up and listen: planar cell polarity regulation in the mammalian inner ear," Seminars in Cell \& Developmental Biology, vol. 20, no. 8, pp. 978-985, 2009.

[38] P. Dallos, "Overview: cochlear neurobiology," in The Cochlea, P. Dallos, A. N. Popper, and R. R. Fay, Eds., vol. 8 of Springer Handbook of Auditory Research, pp. 1-43, Springer, New York, NY, USA, 1996.

[39] T. Abe, S. Kakehata, R. Kitani et al., "Developmental expression of the outer hair cell motor prestin in the mouse," Journal of Membrane Biology, vol. 215, no. 1, pp. 49-56, 2007. 

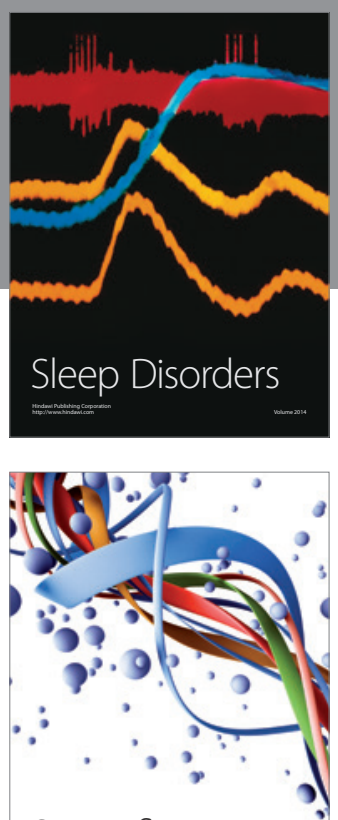

Scientifica
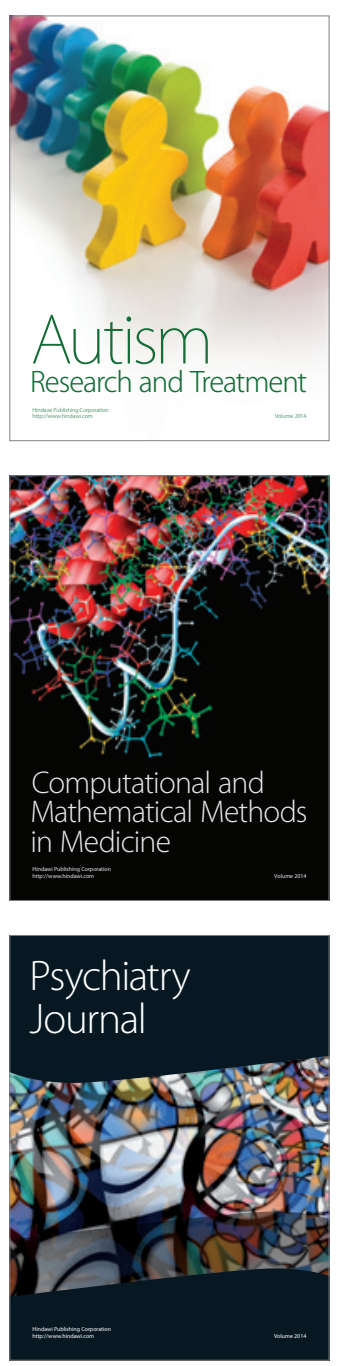
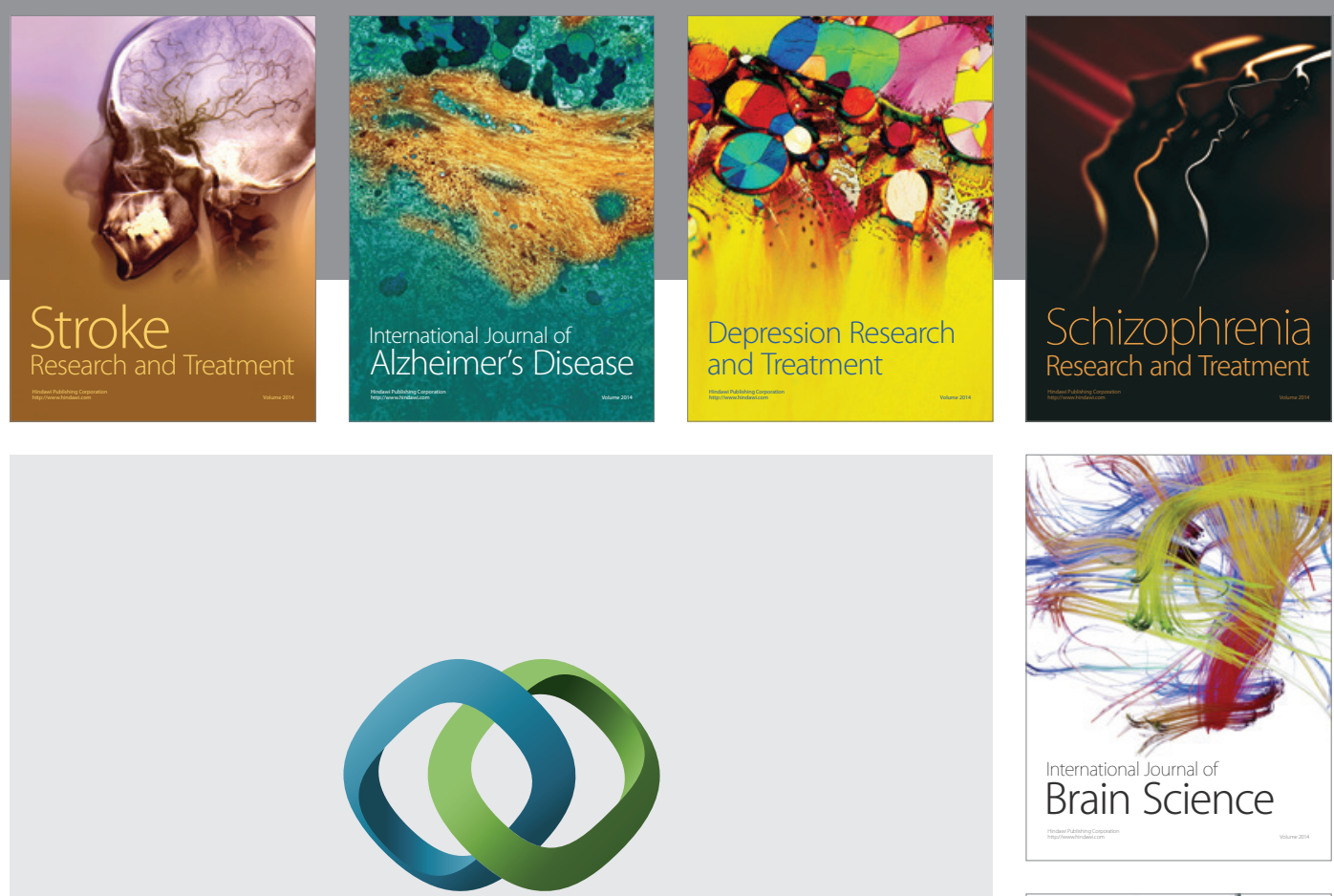

\section{Hindawi}

Submit your manuscripts at

http://www.hindawi.com
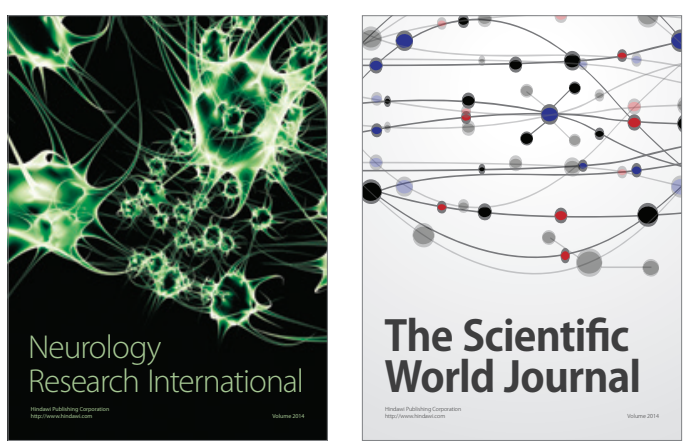

The Scientific World Journal

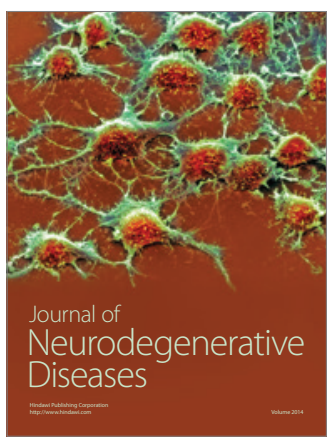

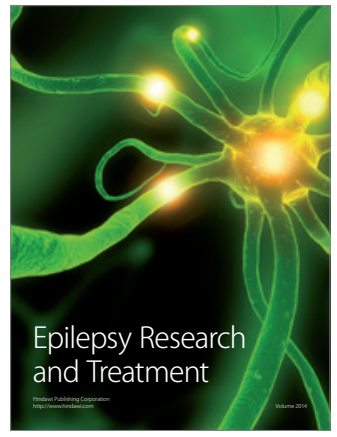

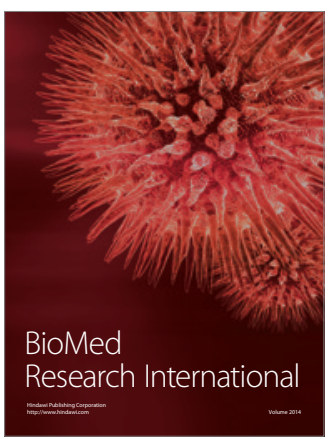

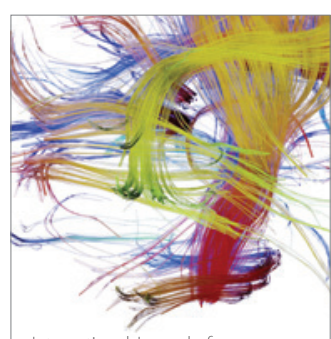

Brain Science

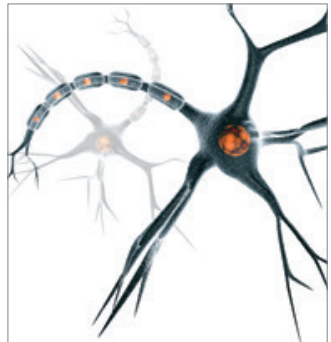

Neural Plasticity
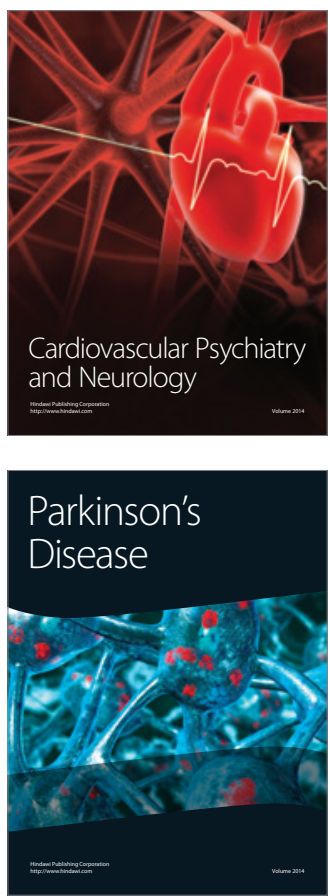